\title{
Early Diagnosis and Prompt Treatment Improves Quality of Life in Patients with Frontal Fibrosing Alopecia
}

\section{Rachita Dhurat Deepti Shukla Ameet Dandale Smita Ghate Sandip Agrawal}

Sujit Shanshanwal

Department of Dermatology, LTMMC and Lokmanya Tilak Municipal General Hospital, Mumbai, India

\section{Established Facts}

- Currently, there is no established definitive therapy for frontal fibrosing alopecia.

\section{Novel Insights}

- Regrowth of hair is possible if intralesional steroid is given in the early stage of frontal fibrosing alopecia.

\section{Keywords}

Frontal fibrosing alopecia $\cdot$ Histopathology $\cdot$ Response to steroid

\begin{abstract}
Frontal fibrosing alopecia (FFA) is a slowly progressive cicatricial alopecia. FFA patients typically present with hair loss in the frontal, temporal, or frontotemporal scalp and eyebrows. It can also involve facial and body hair. It has an early inflammatory stage, which slowly progresses to the stage of fibrosis. In this article, we report a case of FFA in the early inflammatory stage who responded well to intralesional steroid.

2019 S. Karger AG, Basel
\end{abstract}

(C) 2019 S. Karger AG, Basel

\section{Introduction}

Frontal fibrosing alopecia (FFA) is an acquired scarring alopecia presenting as symmetrical recession of frontal and temporal hairline as well as eyebrow loss. This entity was described for the first time by Kossard [1], in 1994, with the term "postmenopausal frontal fibrosing alopecia" (PFFA). It is a clinically distinct variant of lichen planopilaris. This condition no longer exclusively affects postmenopausal women, as small but increasing numbers of cases have been reported in premenopausal women and men; therefore, the term FFA has been suggested instead of PFFA. Pathogenesis of FFA is poorly understood; an autoimmune reaction and hormonal factors seem to play an important role. The natural history of this condition is variable, although slow progression with spontaneous remission is the most frequently reported outcome. Treatment is usually disappointing, al-

Dr. Rachita Dhurat

OPD No. 16, Department of Dermatology

LTMMC and Lokmanya Tilak Municipal General Hospital

Dr. Babasaheb Ambedkar Road, Sion West, Mumbai 400022 (India)

E-Mail rachitadhurat@yahoo.com 
Fig. 1. a-d Bitemporal hair line recession with prominent eyebrow involvement.

Fig. 2. a, b Lonely hair and perifollicular erythema and cast.

Fig. 3. a Atrophy of epidermis. b Perifollicular lymphocytic infiltrate with atrophic sebaceous glands. c Basal cell vacuolization in follicular epithelium.
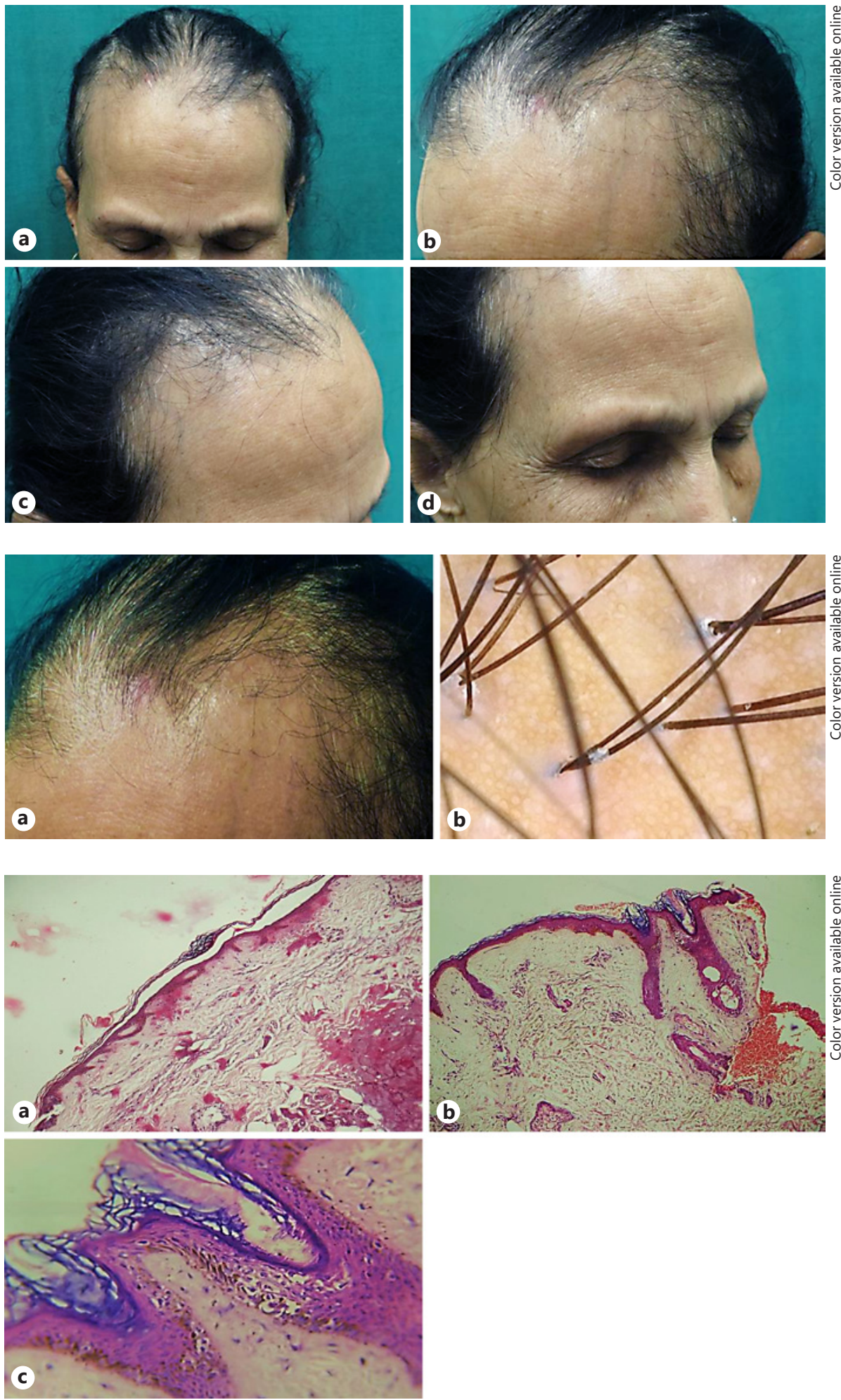

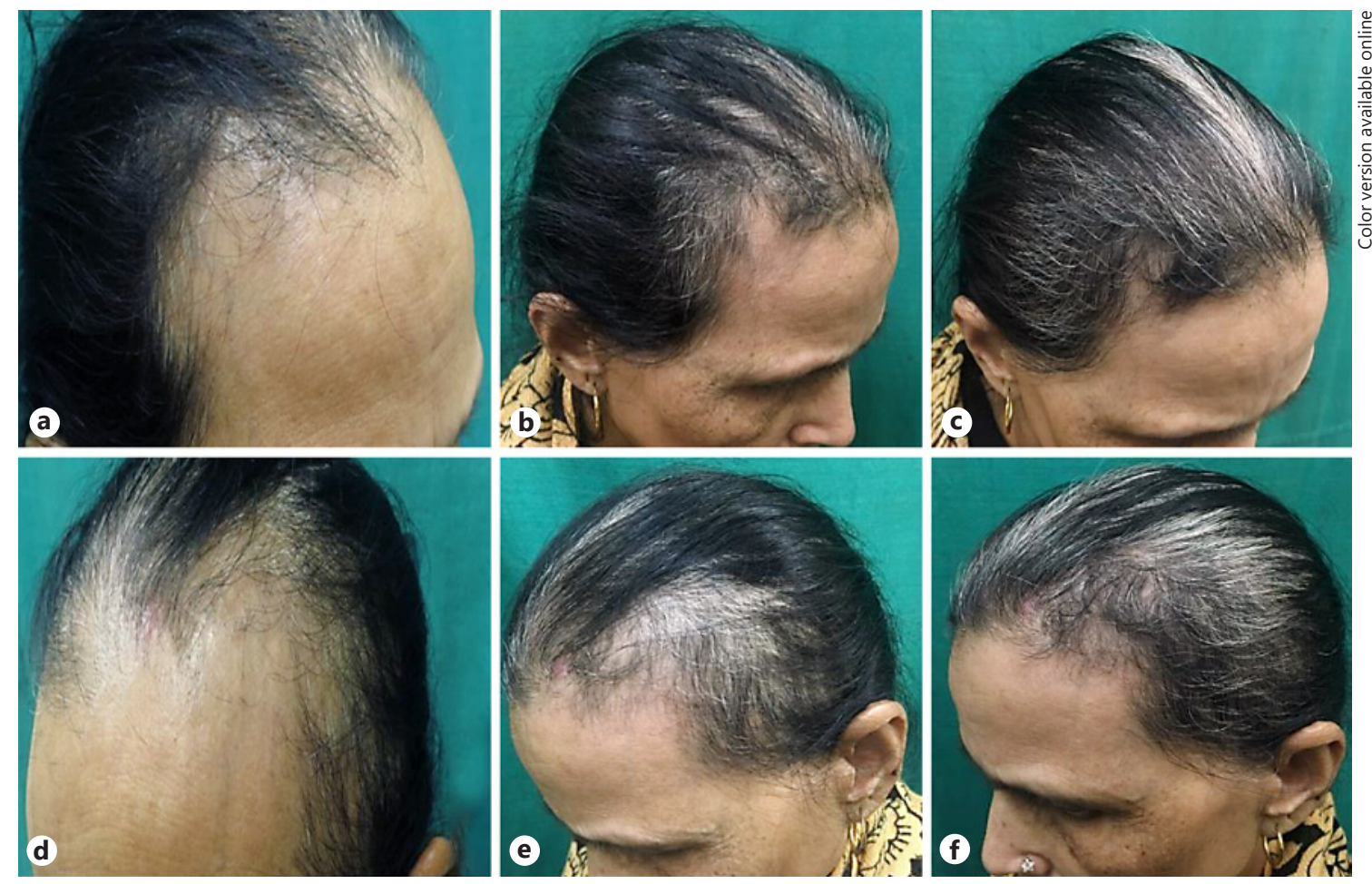

Fig. 4. Posttreatment photographs. a, d First visit. b, e After 2 doses. c, f After 12 weeks of treatment.

though many treatment options like topical and intralesional corticosteroids, antibiotics, hydroxychloroquine, topical and oral immunomodulators, tacrolimus, and 5-alpha-reductase inhibitors are available.

\section{Case Report}

Case 1

A 47-year-old postmenopausal female came with a history of hair loss over the eyebrows and scalp since 1 year. She denied any other disorder of hair or scalp prior to onset of hair loss. Examination showed band-like hair line regression of $2 \mathrm{~cm}$ on the frontal and bitemporal areas. The skin over the affected areas was pale, smooth, and shiny. She also had partial hair loss from both eyebrows (Fig. 1). Hair pull test was negative from the scalp. Body hair and oral and genital mucosae were normal. Trichoscopic examination of the affected scalp revealed markedly reduced follicular ostia, perifollicular erythema, cast, and lonely hair (Fig. 2). Differential diagnoses of alopecia areata and FFA were considered. A 4-mm punch biopsy was done. Histopathological examination of vertical section revealed follicular plugging, diffuse fibrosis in the upper dermis with one follicle showing basal cell vacuolization, and mild perifollicular lymphocytic infiltrate. Atrophic sebaceous glands were noticed. The rest of the epidermis and dermis was unremarkable (Fig. 3). A diagnosis of FFA was made. Intradermal injections of triamcinolone acetate $2.5 \mathrm{mg} / \mathrm{mL}$ were given over bilateral temporal patches and repeated every 4 weeks for 12 weeks. After a single injection, hair regrowth was seen and marked improvement was present at the end of 12 weeks (Fig. 4).

\section{Case 2}

A 57-year-old menopausal woman presented with receding frontal hairline with loss of eyebrows since 10 years. The skin over the alopecia patch was shiny and smooth (Fig. 5). Trichoscopy of the affected scalp revealed loss of follicular openings, perifollicular erythema, and lonely hair (Fig. 6). Histopathological examination of the vertical section of the biopsy sample from the alopecia patch revealed absence of perifollicular inflammatory infiltrate with predominance of perifollicular lamellar fibrosis. Apoptotic keratinocytes in the outer root sheath were seen (Fig. 7). A diagnosis of late-stage FFA was made.

\section{Discussion}

FFA is a primary progressive cicatricial alopecia of the frontal, temporal, or frontotemporal scalp in the absence of follicular keratotic papules over the body along with diffuse bilateral eyebrow alopecia. These features are considered as updated proposed major criteria for the diag- 
Fig. 5. a-c Frontotemporal hair line recession and loss of hair over eyebrows.

Fig. 6. a Perifollicular erythema with scaling. b Loss of follicular opening.

Fig. 7. a Perifollicular lamellar fibrosis with apoptotic keratinocytes (b). c Apoptotic keratinocytes in outer root sheath.

Response to Steroid in the Early Stage of FFA
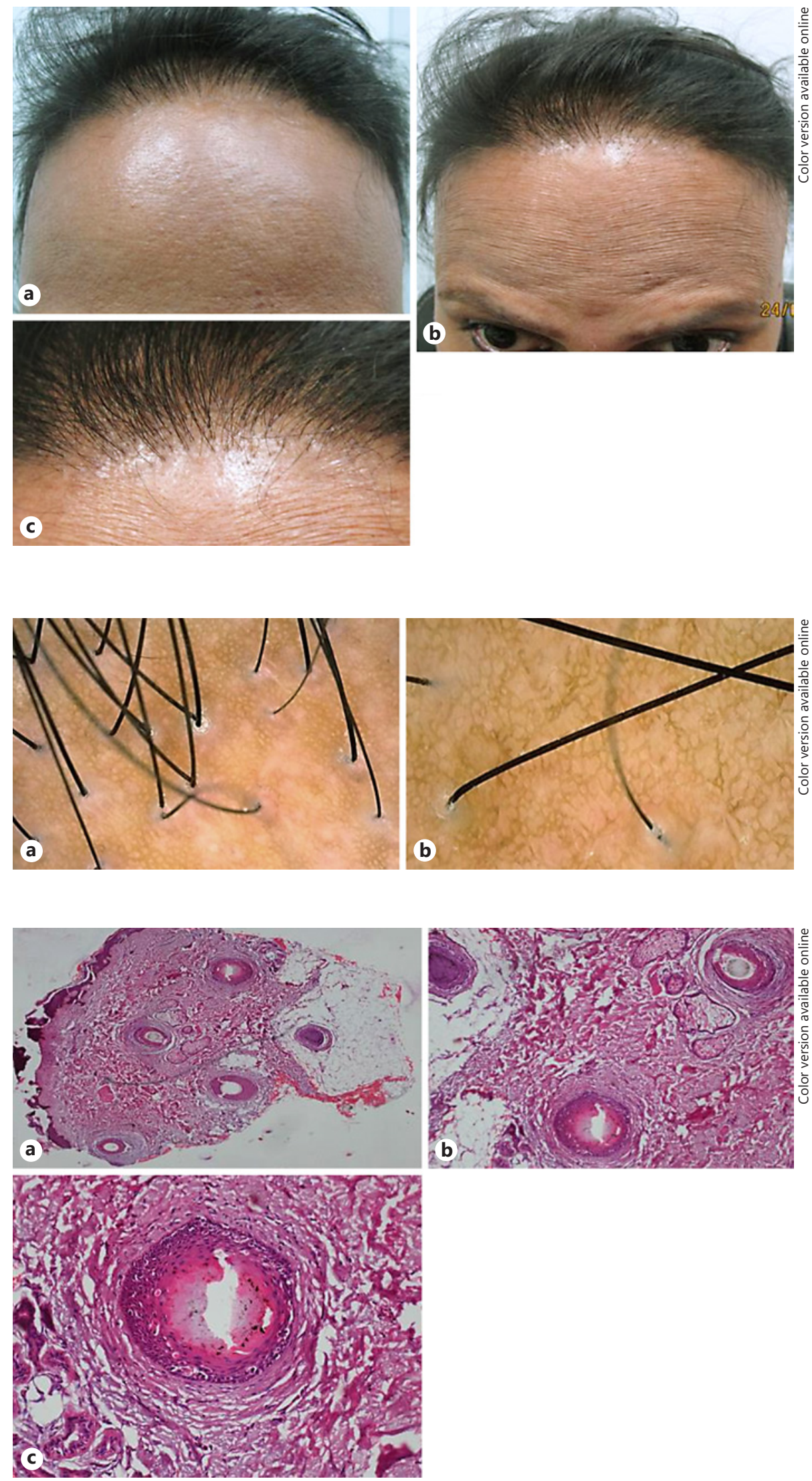

Skin Appendage Disord 2019;5:172-176 DOI: $10.1159 / 000495117$ 
nosis of FFA. Other significant findings like perifollicular erythema, follicular hyperkeratosis, histopathologic features of cicatricial alopecia in the pattern of FFA, involvement of additional FFA sites like occipital area, facial hair, sideburns, or body hair, and noninflammatory facial papules are considered as minor criteria [2]. Characteristic trichoscopic findings of FFA include cicatricial patches, which are areas of absence of follicular openings, perifollicular scaling, and perifollicular erythema. Toledo-Pastrana et al. [3] analysed the frequency of trichoscopic features in 79 patients. They found that $100 \%$ of patients showed absence of follicular opening, $72.1 \%$ showed follicular hyperkeratosis, and $66.3 \%$ showed perifollicular erythema. Understanding histopathological features in the early and late stages would aid in the prognosis of the disease. The early stage of the disease shows predominance of follicular lichenoid lymphocytic inflammatory infiltrate involving infundibulum and isthmus and follicular focal basal vacuolar degeneration, whereas the late stage is characterized by absence of inflammation and predominance of perifollicular lamellar fibrosis [4]. There is currently no established therapy for FFA. Many treatment options like 5-alpha reductase (finasteride and dutasteride), hydroxychloroquine, topical and intralesional steroid, oral doxycycline, and mycophenolate mofetil have been tried by various authors with variable outcome [5]. In a study, Moreno-Ramirez et al. [6] recruited 16 patients for intralesional steroid, given every 3 month for 12 months. All patients showed stabilization of hair line recession. There are very few reports on the use of intralesional steroid in patients with FFA. Herein, we report complete regrowth with intralesional injections of steroid in our first case. The possible explanation to this is that the inflammatory process was arrested leading to prevention of detachment of the arrector pili muscle from the hair follicle. Thereby, stem cells residing in the bulge area retained their potential to regenerate the hair follicle [7]. Our second case reported regression of symptoms and arrest of recession of hair line but no hair regrowth with intralesional steroid. These case reports emphasize of the possibility of regrowth with intralesional steroid given in the early stage of the disease.

\section{Statement of Ethics}

Subjects have given their consent to publish photos and details of their case.

\section{Disclosure Statement}

The authors declare no conflicts of interest.

\section{References}

1 Kossard S. Postmenopausal frontal fibrosing alopecia. Scarring alopecia in a pattern distribution. Arch Dermatol. 1994 Jun;130(6):7704.

2 Vañó-Galván S, Saceda-Corralo D, MorenoArrones ÓM, Camacho-Martinez FM. Updated diag-nostic criteria for frontal fibrosing alopecia. J Am Acad Dermatol. 2018 Jan; 78(1):e21-2.
3 Toledo-Pastrana T, Hernández MJ, Camacho Martínez FM. Perifollicular erythema as a trichoscopy sign of progression in frontal fibrosing alopecia. Int J Trichology. 2013 Jul; 5(3):151-3.

4 Kossard S, Lee MS, Wilkinson B. Postmenopausal frontal fibrosing alopecia: a frontal variant of lichen planopilaris. J Am Acad Dermatol. 1997 Jan;36(1):59-66.

5 Fertig R, Tosti A. Frontal fibrosing alopecia treatment options. Intractable Rare Dis Res. 2016 Nov;5(4):314-5.
6 Moreno-Ramirez D, Camacho Martinez F. Frontal fibrosing alopecia: a survey in $16 \mathrm{pa}-$ tients. J Eur Acad Dermatology Venereol. 2005 Nov; 19(6):700-5.

7 Yazdabadi A, Whiting D, Rufaut N, Sinclair R. Miniaturized Hairs Maintain Contact with the Arrector Pili Muscle in Alopecia Areata but not in Androgenetic Alopecia: A Model for Reversible Miniaturization and Potential for Hair Regrowth. Int J Trichology. 2012 Jul; $4(3): 154-7$ 